\title{
SÍNTESE E CARACTERIZAÇÃO DE PONTOS QUÂNTICOS AMBIENTALMENTE AMIGÁVEIS, UM MEIO SIMPLES DE EXEMPLIFICAR E EXPLORAR ASPECTOS DA NANOCIÊNCIA E NANOTECNOLOGIA EM CURSOS DE GRADUAÇÃO
}

Calink I. L. Santos ${ }^{\text {, Juan C. A. Ferreira }}{ }^{a}$, Letícia R. C. Cunha ${ }^{a}$, Roberto Vaz ${ }^{\mathrm{a}}$ e Marco A. Schiavona,*,(1)

aDepartamento de Ciências Naturais, Universidade Federal de São João del-Rei, Campus Dom Bosco, 36301-160 São João delRei - MG, Brasil

Recebido em 06/12/2019; aceito em 06/04/2020; publicado na web em 29/05/2020

\begin{abstract}
SYNTHESIS AND CHARACTERIZATION OF ENVIRONMENTALLY FRIENDLY QUANTUM DOTS, A SIMPLE WAY TO EXEMPLIFY AND EXPLORE NANOSCIENCE AND NANOTECHNOLOGY IN UNDERGRADUATE EDUCATION. Nanotechnologies and nanomaterials are very current and interesting topics and can be used as interdisciplinary tools in education. They can be used to work in a practical and interesting way with lots of subjects. In chemistry teaching it can be particularly important to consolidate the nanomaterials, nanotechnology and characterization teaching. Among the nanomaterials, quantum dots (QDs) can be highlighted due to their interesting optical and electronical properties. Some of the topics that can be discussed related to them are the nanoparticles synthesis, spectroscopies and optical characterization, X-ray diffractometry and structural characterization, surface ligands, semiconductor materials, electronic transition, solid crystalline structure, colloidal synthesis, among many other subjects. The application of the experiment of synthesis and characterization of environmentally friendly quantum dots to students of the discipline "Solid State Chemistry" of the chemistry undergraduate course, allowed the contact of students with materials of great technological interest. Being a material widely studied by various research groups around the world. The experiment proved to be an interesting way to introduce students to a material of great technological interest, in a simple and accessible way. It aroused the interest of the students while allowing the practical application of the acquired knowledge.
\end{abstract}

Keywords: quantum dots; nanotechnologies; education experiment; solid state chemistry.

\section{INTRODUÇÃO}

A nanotecnologia, que é a ciência que trata do estudo de materiais em escala nanométrica, bem como os nanomateriais, são assuntos bastante interessantes e atuais, ${ }^{1}$ e podem ser usados como ferramentas muito úteis e interdisciplinares na educação, possibilitando trabalhar de maneira prática e de modo interdisciplinar uma diversidade de assuntos. No ensino de química, por exemplo, pode ser particularmente importante correlacionar o ensino de nanomateriais e nanotecnologia a diversos assuntos atuais como: tecnologia, meio ambiente, energia e ainda temas mais específicos como transições eletrônicas, estrutura atômica, síntese em meio coloidal, estrutura de sólidos cristalinos, sínteses de nanopartículas, caracterização óptica, espectroscopias, ligantes de superfície, materiais semicondutores, difração de raios X e caracterização estrutural, bem como diversos outros assuntos.

Entre os nanomateriais, os pontos quânticos (PQs) são materiais que se destacam devido às suas interessantes propriedades ópticas e eletrônicas, ${ }^{2}$ que dão a esses materiais aplicações em muitos campos, e, portanto, os tornam bastante interessantes do ponto de vista tecnológico. ${ }^{3}$ Os pontos quânticos são semicondutores nanocristalinos, que apresentam portadores de carga (elétrons e buracos) em um estado de forte confinamento, chamado de confinamento quântico, devido às suas dimensões físicas (entre 1 e $10 \mathrm{~nm}$ ), menores que o raio de Borh do éxciton. ${ }^{4}$ Esse confinamento é responsável pela absorção e emissão na região do visível e regiões próximas (como o ultravioleta e o infravermelho próximo), apresentadas por esses materiais. ${ }^{5}$

Uma característica bastante interessante, também apresentada, é a relação das propriedades ópticas com o processo sintético, de forma que uma certa propriedade desejada pode ser alcançada por

*e-mail: schiavon@ufsj.edu.br meio de ajustes no procedimento de síntese. ${ }^{6}$ Sendo uma característica marcante desses materiais, portanto, a dependência de suas propriedades de absorção e emissão com seus tamanhos. ${ }^{7}$ Dessa forma, alterações sensíveis no tamanho das nanopartículas podem ser responsáveis por modificações na energia de transição e, consequentemente, alterações nas propriedades ópticas, conforme pode ser observado na Figura 1.

De forma geral, quando o tamanho físico do semicondutor é reduzido abaixo do Raio de Bohr do éxciton, que é a distância média entre o elétron e o buraco, ocorre o efeito chamado de confinamento quântico, uma vez que a natureza quântica do éxciton é exposta ao tamanho do cristal. ${ }^{8}$ De forma qualitativa, o confinamento quântico leva à formação de níveis discretos nas bandas de valência e de condução desses materiais, que apresentam então comportamento intermediário entre cristais e átomos isolados. O confinamento dos pares de elétrons e buracos nas três dimensões leva ao aumento na energia entre a banda de valência e a banda de condução (energia de banda proibida - bandgap) dos materiais, de acordo com a diminuição de seu tamanho, ou seja, quanto menor o material, maior o confinamento dos portadores de carga, e maior a sua energia de banda proibida. ${ }^{9}$ Consequentemente, a absorção e emissão ópticas dos materiais tendem a serem dependentes de seus tamanhos e, assim, a se deslocarem para a região do azul do espectro eletromagnético à medida que seus tamanhos vão se tornando cada vez menores, conduzindo a um arco-íris de cores de emissão, da região do ultravioleta ao infravermelho (representado na Figura 1).

Devido a essas propriedades, PQs apresentam aplicação em uma diversidade de áreas, por exemplo, como absorvedores em dispositivos conversores de energia solar, ${ }^{10}$ emissores de luz, ${ }^{11}$ biomarcadores, ${ }^{12}$ sensores fluorimétricos ${ }^{13}$ e muitos outros, ${ }^{14,15}$ sendo considerados materiais interessantes para a nanotecnologia, ao mesmo tempo em que podem ser usados para trabalhar muitos tópicos em educação. 
Pontos Quânticos

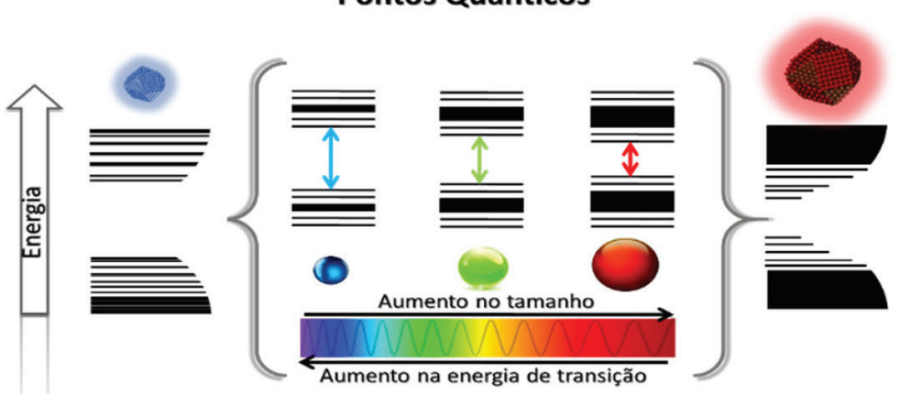

Cristal

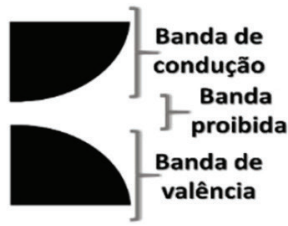

Figura 1. Representação do efeito de variação da energia de banda proibida em função do tamanho da nanopartícula

PQs são divididos de acordo com os diferentes grupos da Tabela Periódica que seus elementos constituintes pertencem, como, IV-VI (sulfeto de chumbo, PbS), II-VI (telureto de cádmio, CdTe) e III-V (fosforeto de gálio, GaP). ${ }^{16}$ Em diversas metodologias de síntese, metais tóxicos são utilizados, como $\mathrm{Cd}$ e $\mathrm{Pb}$, o que leva algumas das classes de PQs a serem consideradas tóxicas, dificultando também suas aplicações. ${ }^{17,18}$ PQs ternários II-III-VI, por outro lado, são comumente tratados como materiais "verdes", ou ambientalmente amigáveis, uma vez que não possuem metais tóxicos como constituintes. ${ }^{19}$ Tanto a redução na toxicidade quanto a simplicidade sintética os tornam ainda mais interessantes para aplicação.

Pontos quânticos ternários do tipo II-III-VI comumente apresentam estrutura do tipo calcopirita. ${ }^{20-22} \mathrm{O}$ aumento na complexidade da estrutura dos PQs ternários faz com que eles apresentem, além da dependência entre as propriedades ópticas e o tamanho das partículas, uma dependência entre essas propriedades e a composição do material. ${ }^{23}$ Dessa forma, sensíveis alterações nos ângulos de ligação, e portanto distorções da estrutura, geradas por alterações estequiométricas, possibilitam também a modificação das propriedades ópticas dos materiais. ${ }^{24,25}$ Assim, a proporção molar entre os precursores é considerada um parâmetro bastante importante na síntese.

Outro tópico importante sobre os pontos quânticos são as técnicas utilizadas em sua caracterização e como elas podem ser usadas para ensinar na graduação, principalmente devido à possibilidade de utilização de técnicas relativamente simples, que são tópicos no ensino de química, como espectroscopias, difração de raios $\mathrm{X}$ e análises relacionadas ao estudo da composição e morfologia dos materiais.

Uma vez que o tamanho da partícula é uma propriedade de grande influência para pontos quânticos, é importante que se tenha um certo controle dessa propriedade. Dessa forma, com o intuito de controlar o tamanho, bem como evitar agregação e possibilitando ainda a funcionalização dos PQs, são utilizados ligantes de superfície. ${ }^{26} \mathrm{O}$ papel do ligante é passivar a superfície dos nanocristais, coordenando-se por meio de ligações erráticas (não completas), na superfície dos PQs. A passivação se dá pela interação com orbitais desacoplados de átomos superficiais, possibilitando a redução de estados de armadilha de elétrons da superfície. ${ }^{27} \mathrm{~A}$ passivação pode, portanto, melhorar as propriedades relacionadas a defeitos de superfície, levando assim ao aumento do rendimento quântico de fluorescência do material, $\phi_{\mathrm{f}}$, sendo essa uma medida da probabilidade do processo de desativação dos estados excitados ocorrerem por fluorescência, e portanto relacionada à capacidade de emissão do material. ${ }^{28}$

Dentre os métodos de preparação dos pontos quânticos, a síntese em um sistema coloidal aquoso se destaca por ser um método que além de possuir uma maior simplicidade experimental, possui boa reprodutibilidade sintética e mais baixo custo. É considerado ainda menos tóxico e possibilita a obtenção de nanopartículas que se dispersam em água, representando, portanto, uma alternativa viável para fabricação de materiais aplicáveis a custos mais baixos. ${ }^{29}$
Apesar de apresentar inúmeras vantagens, sínteses em meio aquoso normalmente produzem nanocristais com valores de rendimento quântico mais baixos que rotas em meio orgânico, que chegam a ter rendimentos de até $80 \% .{ }^{30}$ Uma alternativa utilizada para se melhorar o rendimento quântico em PQs é baseada no crescimento de uma camada externa, composta por uma matriz inorgânica, sobre a superfície dos nanocristais, resultando em estruturas do tipo caroço-casca, conhecidas como, sistemas core-shell. ${ }^{31}$ Tais sistemas são formados por uma combinação de dois semicondutores distintos, em que um deles permanece no centro, core, sendo recoberto por uma camada contendo outro tipo de semicondutor, shell. Nesses sistemas, além de se elevar o rendimento quântico de fotoluminescência, tem-se também um ganho de estabilidade dos nanocristais e, ainda, pela escolha adequada dos materiais do caroço e da casca é possível estender a emissão desses nanomateriais para uma faixa mais ampla do espectro visível. ${ }^{32}$

Dentre os PQs, aqueles que possuem composição baseada no uso de cobre, índio, e enxofre, por exemplo, representam uma alternativa promissora frente a sistemas do tipo II-VI (como CdTe), apresentando bandgap direto e na região do visível, alto coeficiente de absorção, ${ }^{33}$ e ainda alta fotoestabilidade. ${ }^{34}$ Pontos quânticos de sulfeto de cobre e índio (CIS) e semelhantes, como o sulfeto de cobre índio e zinco (CIZS), apresentam absorção e emissão desde a região do infravermelho próximo à região do visível, sendo ainda considerados materiais mais "seguros", eco-amigáveis e de menor toxicidade que materiais contendo metais tóxicos. ${ }^{35}$ Referindo-se mais especificamente ao CIZS, o uso do zinco na composição dos PQs possibilita, além de reduzir a quantidade necessária de índio, melhorar as propriedades relativas à capacidade de emissão do material, bem como deslocar a emissão para regiões de maior energia. Dessa forma, a abordagem de sínteses, desses materiais, cada vez mais simples e com impactos ambientais reduzidos, bem como métodos que possibilitem o controle das suas propriedades são tópicos bastante abordados.

Diversos conceitos relacionados aos pontos quânticos e à nanotecnologia podem ser usados como ferramentas no ensino de química. Nesse trabalho é proposta a preparação e caracterização de pontos de quânticos de sulfeto de cobre, índio e zinco (CIZS) em meio aquoso, bem como sulfeto de cobre, índio e zinco com uma casca de $\mathrm{ZnS}$ (CIZS/ZnS), em aula prática, de Química Inorgânica ou Química do Estado Sólido, para a graduação em química. A síntese utiliza uma metodologia simples e que possibilita obtenção de nanocristais de qualidade e com potencial para diversas aplicações. ${ }^{36}$ Visa-se com a utilização desse experimento possibilitar a motivação de alunos de graduação em química, pelo contato direto com a obtenção de nanomateriais de interesse para várias aplicações tecnológicas, bem como o contato com as caracterizações e conceitos envolvidos à nanociência e técnicas de análises.

Nas sessões a seguir são apresentados alguns conceitos e conteúdos que podem ser abordados durante a experimentação envolvida 
na produção e caracterização de pontos quânticos ambientalmente amigáveis de sulfeto de cobre, índio e zinco.

\section{Toxicidade relacionada aos metais}

Metais tóxicos estão distribuídos no meio ambiente por diversos meios. Alguns dos processos que geram suas liberações são erupções vulcânicas, erosão, atividade bacteriana e sobretudo por meio de atividades antropogênicas, como a combustão de combustíveis fósseis, processos industriais e até atividades agrícolas.

Esses metais se bioacumulam em organismos vivos e no corpo humano, causando diversos efeitos. No corpo humano, por exemplo, esses metais são transportados e compartimentados em células e tecidos, ligando-se a proteínas, ácidos nucleicos, destruindo essas macromoléculas e interrompendo funções celulares, apresentando várias consequências para o corpo. ${ }^{37}$

Algumas das possíveis consequências são: afetar o sistema nervoso central, levando a distúrbios mentais, bem como doenças relacionadas aos pulmões, sangue, fígado, rins, e outros órgãos vitais. A acumulação de metais a longo prazo pode também levar à progressão de processos degenerativos físicos, musculares e neurológicos, semelhantes a doenças como Parkinson e Alzheimer. O contato prolongado pode ainda levar a alterações hormonais, atingindo o sistema endócrino e reprodutivo, causando mutações e levando ao câncer. Essas são algumas das consequências diretas do contato com metais tóxicos, podendo esses serem responsáveis por uma diversidade de danos ambientais. ${ }^{38}$

\section{Calcopirita - características do cristal}

Descrita em 1954, por Goodman e Douglas, ${ }^{39}$ como um tipo ternário da estrutura do diamante, a calcopirita é considerada um semicondutor, sendo caracterizada pela regra de Grimm-Sommerfeld como pertencente à classe IV de semicondutores. ${ }^{40}$ Nessas estruturas, cada átomo apresenta número de coordenação 4 , sendo esses conectados por ligações $\mathrm{sp}^{3} \mathrm{e}$ arranjados em um tetraedro.

Compostos ternários de calcopirita podem ser considerados uma variação de estruturas binárias da blenda de zinco. Em comparação com a blenda de zinco, no entanto, uma redução de simetria ocorre devido à presença de dois ou mais tipos de cátions, levando a uma célula primitiva composta pelo dobro de átomos na calcopirita, em relação à célula primitiva da blenda de zinco (Figura 2).

(a)

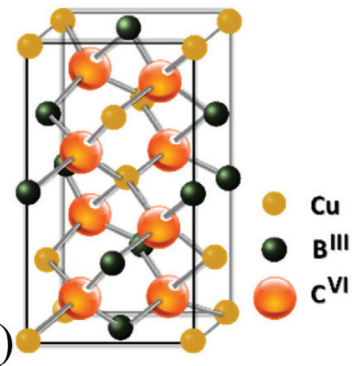

(b)

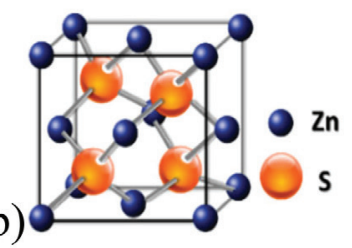

Figura 2. Representação esquemática de células unitárias de calcopirita (a) e blenda de zinco $(b)$

Pequenas mudanças na estrutura podem gerar alterações na energia da banda proibida, podendo, no caso dos semicondutores, estas mudanças serem produzidas por dois importantes fatores. O primeiro fator pode estar relacionado de forma puramente eletrônica, sendo causado por estados p-d repulsivos, na formação da banda de valência, que está associada aos orbitais d do átomo de cobre, que devido a processos de oxidação pode apresentar valências mistas. Já o segundo fator, segundo
Jaffe et al.,${ }^{24}$ é um simples parâmetro estrutural, relacionado à posição do ânion no eixo x, causado pelas diferenças entre as ligações do ânion com os diferentes cátions. Dessa forma, o sensível posicionamento do ânion pode "controlar" o bandgap e o comportamento óptico desses materiais, levando calcopiritas que exibem caráter d semelhantes a apresentarem diferentes valores de bandgap, uma vez que apresentam diferentes tamanhos de ligação do ânion com os diferentes cátions, sendo que, em estruturas com caráter d semelhantes, um aumento na distorção do tetraedro pode levar a um menor valor de bandgap..$^{25}$ Esses efeitos são ainda mais pronunciados em materiais em escala nanométrica, como os pontos quânticos, de forma que uma forte influência desses fatores pode ser exercida sobre esses materiais.

\section{Análises espectroscópicas}

A espectroscopia é o estudo da interação da radiação eletromagnética com a matéria. De acordo com o fenômeno físico-químico e a energia de radiação, diferentes espectroscopias são classificadas. Essas espectroscopias são tópicos particularmente importantes no estudo e ensino de química, trazendo importantes conceitos de química, física e análise. Algumas das principais espectroscopias utilizadas na caracterização de PQs são apresentadas na sequência.

\section{Espectroscopia eletrônica de absorção, no UV-vis, e de emissão}

Uma espectroscopia particularmente importante para a análise de pontos quânticos é a espectroscopia eletrônica, na região do ultravioleta e do visível. Átomos e moléculas podem absorver fótons (partículas de radiação eletromagnética) e, portanto, absorver sua energia, levando elétrons de valência a estados excitados. Essa movimentação de elétrons entre estados de diferentes energias, tanto de um nível mais baixo para um mais alto, quanto no sentido inverso, é chamada de transição. A transição eletrônica possui valores energéticos específicos (quantização), variando de material para material, sendo necessário, para que ocorra a transição, que o fóton possua energia maior que a energia referente à diferença de energia entre os níveis energéticos envolvidos. Essas transições podem ser mais bem visualizadas no diagrama de Jablonski. ${ }^{41,42}$

A energia de transição é única e diferente para cada átomo, de forma que, no caso de átomos, por exemplo, é possível a identificação por meio do espectro de emissão. Da mesma forma, para pontos quânticos, importantes informações podem ser obtidas tanto por meio do espectro de emissão quanto de absorção, uma vez que as propriedades ópticas desses materiais são uma das características mais importantes apresentadas por eles. Dado que PQs apresentam relação entre o tamanho da partícula e a energia de transição, é possível inferir por exemplo, por meio do espectro de absorção se o material cresce, com variações feitas durante a síntese, ${ }^{43}$ sendo possível ainda estimar a energia de banda proibida e propriedades relativas à região e capacidade de emissão dos materiais.

\section{Espectroscopia na região do infravermelho}

Assim como na espectroscopia UV-vis, em que se estuda o comportamento do material por meio de suas transições eletrônicas quando é feita a excitação com fótons, quando é utilizada radiação com energia mais baixa, como na região do infravermelho do espectro eletromagnético, é possível também obter informações provenientes das mudanças que ocorrem em átomos e moléculas. Considerando-se que a energia envolvida nessa região não é suficiente para gerar uma transição eletrônica (excitar os elétrons para diferentes níveis), outro tipo de comportamento é gerado. A radiação na região do infravermelho gera a vibração das ligações químicas, que se "movimentam" de diferentes formas. Cada ligação possui energia específica de vibração, para cada forma de vibração, assim como as transições eletrônicas. ${ }^{44}$ 
Assim, a espectroscopia vibracional é baseada nas vibrações individuais de átomos, em moléculas, em relação a átomos vizinhos, quando estes interagem com fótons, considerando-se uma posição de equilíbrio. Ligações entre átomos podem sofrer vibrações tanto com movimentos axiais quanto angulares (podendo sofrer alongamento, flexão ou torção acerca da posição de equilíbrio). A inspeção dos espectros de infravermelho dos materiais possibilita obtenção de informações a respeito das ligações presentes. ${ }^{43} \mathrm{Em}$ pontos quânticos, a espectroscopia na região do infravermelho pode gerar informações referentes aos ligantes de superfície, indicando por exemplo uma possível posição de interação entre o ligante e o nanocristal, bem como a sua presença na superfície do material.

\section{Difração de Raios X}

Outros tópicos importantes que podem ser estudados usando PQs são as estruturas de sólidos e a difração de raios X. A difratometria de raios $\mathrm{X}$ é uma das técnicas de análise mais importantes quando se trata de materiais cristalinos, sendo amplamente utilizada na determinação de fases cristalinas em uma diversidade de materiais. A caracterização por meio da difratometria de raios $\mathrm{X}$ é possível devido à presença de planos ordenados observada em cristais.

Quando há incidência de raios X sobre uma amostra, a radiação de alta energia interage com os átomos presentes, levando ao fenômeno conhecido como difração. Essa difração ocorre como descrito pela Lei de Bragg (Equação 1), cumprindo a relação entre os ângulos de difração e a distância entre os planos referentes (característicos das fases cristalinas). ${ }^{45}$

$$
n \lambda=2 d \operatorname{sen}(\theta)
$$

Considerando-se um pó cristalino, que contém um grande número de pequenos cristais orientados, quando a amostra é colocada na direção de um feixe monocromático de raios $\mathrm{X}$, ocorrerá difração nos planos em que os cristalitos se encontram orientados, em ângulos que cumpram a condição de Bragg. Dessa forma, conhecendo-se os planos específicos de um material, é possível obter informações sobre a sua estrutura. Além disso, é possível explorar o formato dos difratogramas para nanopartículas, que apesar de cristalinas, apresentam picos poucos intensos e alargados devido à presença de poucos planos, que ocorrem como consequência do efeito do tamanho reduzido das nanopartículas. ${ }^{46}$

\section{Estudo da composição e morfologia dos materiais}

Informações bastante importantes quando se trata da caracterização de materiais estão relacionadas à composição e à morfologia. Uma técnica comumente utilizada na determinação dessas propriedades, apesar da disponibilidade limitada, é a microscopia eletrônica de transmissão. O microscópio eletrônico possibilita a obtenção de imagens das amostras em escala nanométrica, sendo possível obter informações referentes ao tamanho das partículas, por exemplo, algo particularmente importante quando se trata de pontos quânticos.

A análise é realizada por meio da incidência de um feixe de elétrons, esse feixe passa pela amostra sofrendo uma série de interações, dependentes das características do material. Dependendo das interações sofridos pelos elétrons, diferentes imagens são formadas. A incidência do feixe de elétrons, de alta energia, permite a obtenção de imagens de alta resolução, possibilitando obter informações diversas, como, estrutura cristalina, deslocamento e limites de grãos, composição, forma, tamanho, bem como o estudo do crescimento de camadas, sendo uma ferramenta bastante "poderosa" para as ciências de materiais. ${ }^{47}$
Diversas outras técnicas possibilitam a obtenção de informações referentes à morfologia ou à composição de materiais, como a espectroscopia de fotoelétrons excitados por raios X (XPS), no entanto, a disponibilidade dessas técnicas pode ser ainda mais limitada.

\section{Ligantes de superfície}

Ligantes de superfície são normalmente compostos orgânicos, contendo pelo menos um grupo de ancoragem (meio orgânico), para interação com a superfície dos PQs. Quando utilizados em meio orgânico, possuem uma longa cadeia de hidrocarbonetos, que interage com o meio apolar, enquanto em meio aquoso possuem cadeia carbônica curta, e mais de um grupo polar, de forma que possam interagir com a superfície dos PQs e com o meio.

Um exemplo comum de ligante de superfície utilizado em meio apolar é o dodecanotiol (DDT), Figura 3, que possibilita então a passivação e controle do crescimento de PQs em meio orgânico. Já para o meio polar, alguns exemplos comuns são o ácido tioglicólico (TGA), o ácido mercaptopropiônico (MPA) e a glutationa reduzida (GSH), representados na Figura 4, em que é possível a observação de pelo menos dois grupos polares, na estrutura de cada ligante, possibilitando a dispersão das nanopartículas em água.
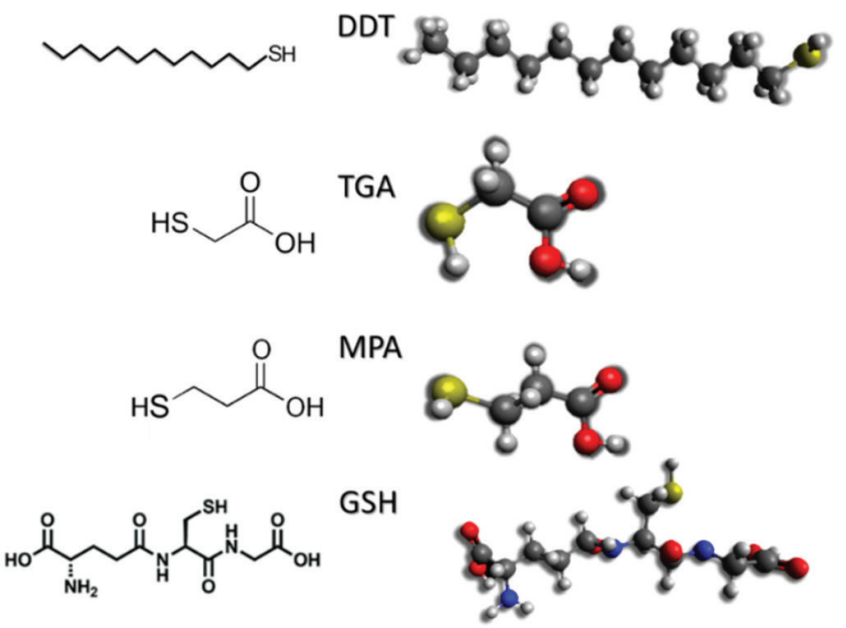

Figura 3. Representação bidimensional e tridimensional dos ligantes DDT, TGA, MPA e GSH

É importante destacar que para o uso dos ligantes em meio aquoso é necessário fazer o controle do $\mathrm{pH}$ do meio reacional, uma vez que em meio com $\mathrm{pH}$ superior ao relacionado às constantes de dissociação ácida (Ka) dos grupos, é esperado que os grupos se encontrem desprotonados.$^{43}$ Isso possibilita a interação tanto com os PQs quanto com o solvente (água). A relação de desprotonação dos ligantes TGA, MPA e GSH com o pH é apresentada na Figura 4.

Sendo o enxofre menos eletronegativo que o oxigênio, tióis não fazem ligações de hidrogênio fortes, e consequentemente, têm atrações intermoleculares mais fracas, não são muito bem solvatados em solventes próticos (como a água), podendo ser bons nucleófilos ${ }^{48} \mathrm{e}$ interagirem mais facilmente com ácidos moles, sendo possivelmente a extremidade que interage com os PQs. Representações ilustrativas dos PQs, tanto com ligante de cadeia longa (para meio orgânico), quanto com ligante de cadeia curta (para meio aquoso, com e sem solvatação), são apresentadas na Figura 5.

\section{Rendimento quântico de fluorescência}

Quando um fluoróforo absorve um fóton de luz, um estado 


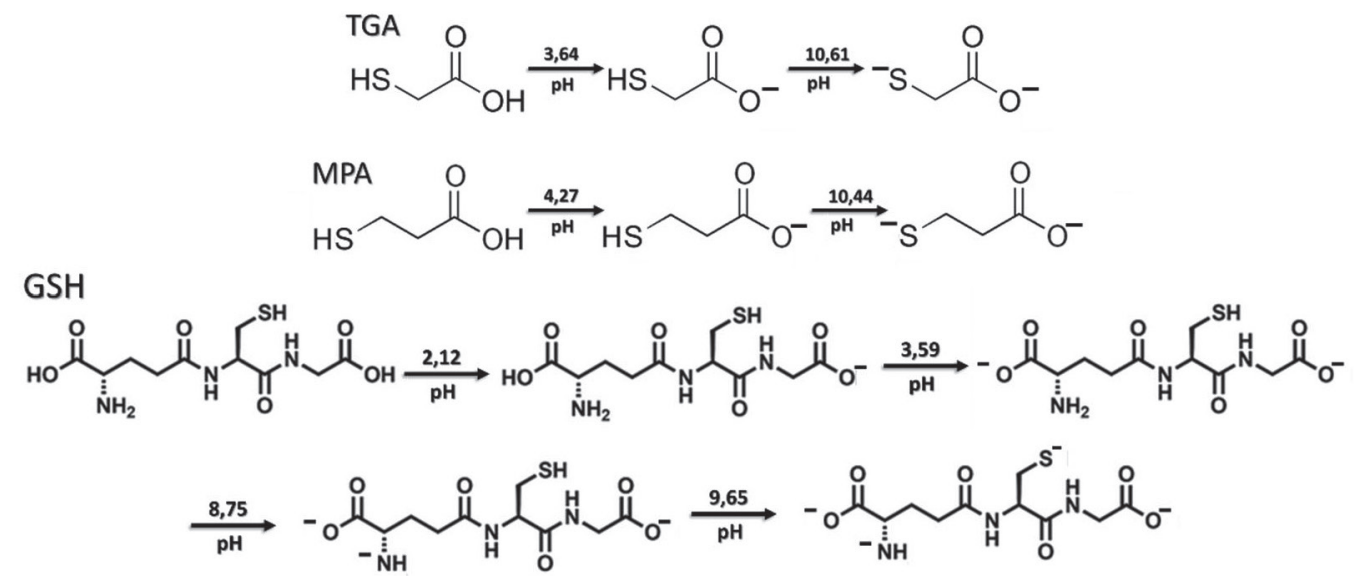

Figura 4. Representação da desprotonação dos ligantes TGA, MPA e GSH, relacionados ao pH
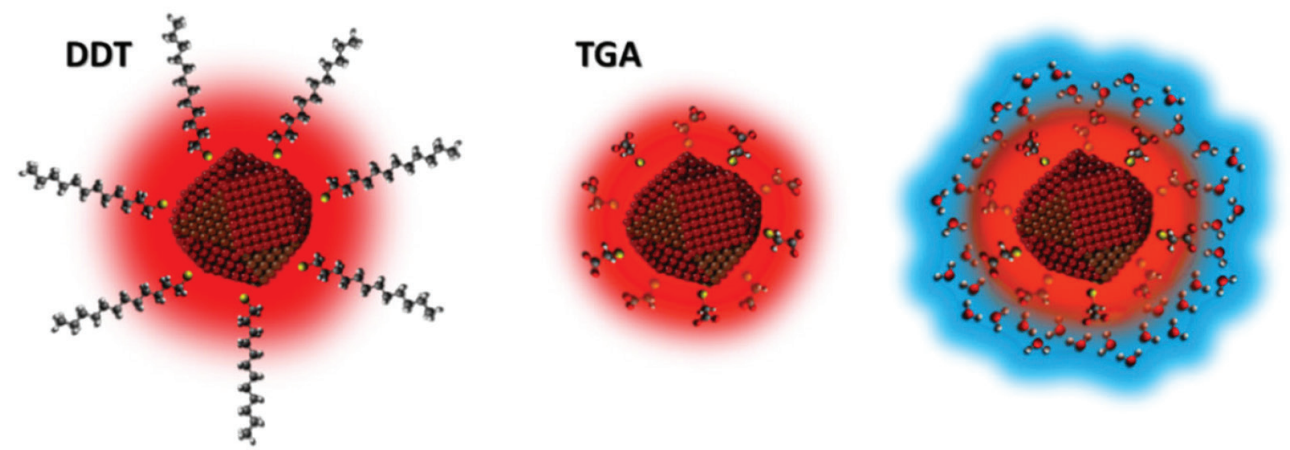

Figura 5. Representação dos $P Q$ s estabilizados com os ligantes DDT e TGA (sem e com solvatação, respectivamente)

excitado é formado, resultando em posterior desativação e, portanto, liberação de energia. Os principais processos de desativação são, a fluorescência (em que a liberação de energia ocorre pela emissão de fótons), conversão interna e relaxação vibracional (em que estão envolvidos processos não-radiativos, como perda de calor para o meio) e cruzamento inter-sistemas (também com decaimento não-radiativo).

Uma medida muito utilizada na caracterização de materiais fotoluminescentes, a razão entre os fótons absorvidos e os fótons emitidos por fluorescência, conhecida como rendimento quântico de luminescência, mede a probabilidade de o processo de desativação dos estados excitados ocorrerem por fluorescência. Para o cálculo do rendimento quântico de fluorescência é comum o uso do método comparativo de Williams et al., ${ }^{49}$ em que se utiliza como referência uma amostra padrão, com rendimento conhecido.

O cálculo do rendimento quântico de fluorescência é feito por meio da Equação 2, utilizando-se o rendimento conhecido de uma amostra padrão e o gradiente dos gráficos obtidos plotando-se a relação entre a absorbância (no comprimento de onda de excitação) e a área integrada de fotoluminescência, da amostra e do padrão.

$$
\phi_{x}=\phi_{P}\left(\frac{\operatorname{Grad}_{x}}{\operatorname{Grad}_{p}}\right)\left(\frac{\eta_{x}^{2}}{\eta_{p}^{2}}\right)
$$

em que $\phi_{x}$ é o rendimento quântico da amostra, $\phi_{P}$ é o rendimento conhecido da amostra padrão, Grad $_{x}$ é a inclinação da curva da área de fotoluminescência integrada em função da absorção, para a amostra e $\operatorname{Grad}_{p}$ para o padrão. $\eta$ é o índice de refração do solvente, sendo o $\eta_{x}$ referente à amostra e $\eta_{p}$ ao padrão.

\section{Síntese coloidal}

São chamados de coloides os sistemas compostos por duas ou mais fases, em que uma dessas é uma fase contínua, chamada de dispersante, e as demais fases são descontínuas, ditas fases dispersas, que possuem tamanhos da ordem de $1 \mathrm{~nm}$ a $1 \mu \mathrm{m}$. Dispersões coloidais são consideradas sistemas intermediários entre as soluções (sistemas homogêneos, com fase dispersa de ordem molecular) e sistemas heterogêneos (com partículas de tamanhos superiores a $10 \mu \mathrm{m}$ ).$^{50}$

A síntese coloidal de nanopartículas inorgânicas se trata de uma reação de precipitação que ocorre em uma fase contínua líquida. A fase sólida apresenta alto grau de dispersão das partículas, com baixa taxa de aglomeração e sedimentação. Em sínteses coloidais, dois são os processos principais, a nucleação e o crescimento das partículas, que ocorrem de forma praticamente simultânea. A nucleação é o processo inicial, em que ocorre a formação de uma fase termodinamicamente distinta, composta por um pequeno número de átomos, íons ou moléculas, que se organizam em um padrão característico de um sólido cristalino, em que partículas adicionais são depositadas à medida que o cristal cresce. Inúmeros dos cristalitos formados apresentam equilíbrio instável com a fase contínua e são depositados, levando ao crescimento das partículas. ${ }^{51}$

Uma reação de precipitação sem o controle dos processos de nucleação e crescimento pode levar à aglomeração descontrolada das partículas e, portanto, à formação de um precipitado com dimensões superiores à dos coloides. Dessa forma, para a obtenção de uma precipitação coloidal é imprescindível a estabilização de superfície, de forma que possa ser controlada a agregação e o crescimento dos materiais. ${ }^{50}$

\section{Estruturas do tipo casca/caroço ("core/shell")}

A química de superfície de PQs apresenta um papel muito importante em suas propriedades, sendo de grande influência no rendimento quântico e na estabilidade. A presença de defeitos de superfície, por 

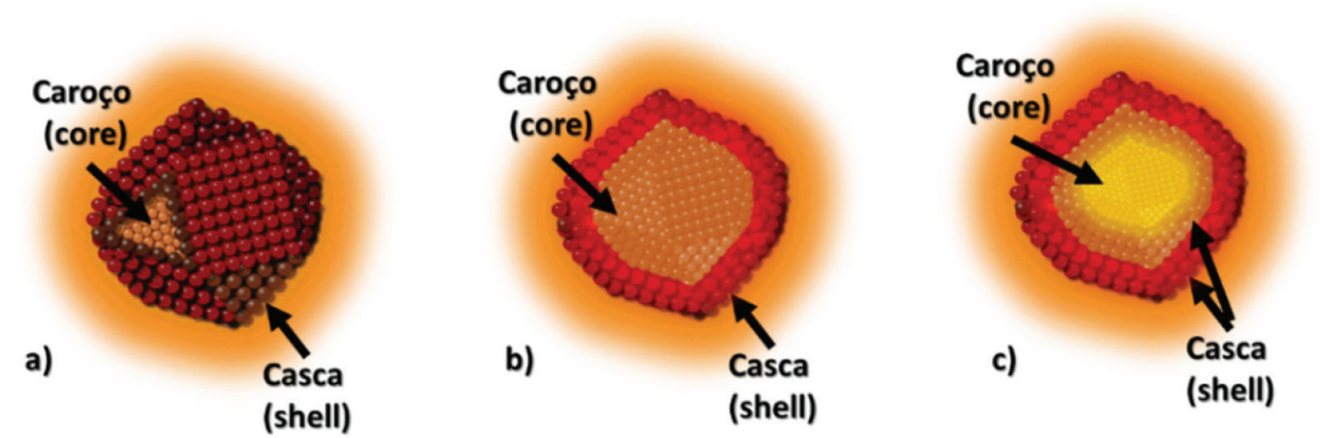

Figura 6. Esquemas de estruturas do tipo caroço/casca, representação tridimensional (a) e em um corte bidimensional, com uma camada de casca (b) e com duas camadas de casca (c)

exemplo, é comumente relatada como motivo de perda de parte da energia emitida por meio de decaimentos não radiativos, causando a diminuição do rendimento quântico de fluorescência. ${ }^{52}$

Um meio de diminuir os defeitos de superfície nesses semicondutores é o crescimento de uma casca (shell), de outro material semicondutor, sobre os PQs, ${ }^{53}$ formando as estruturas chamadas de core/shell ou caroço/casca (Figura 6). O crescimento de uma casca de um semicondutor distinto na parte externa dos PQs possibilita, além da elevação no rendimento quântico de fluorescência, aumentar a estabilidade dos materiais, possibilitando até mesmo reduzir a toxicidade do material do caroço se o crescimento da casca for feito com um material menos tóxico, que vai manter o material do caroço isolado, diminuindo a toxicidade final do material preparado. ${ }^{54}$

Sistemas caroço/casca podem ser sintetizados por meio de diversos procedimentos experimentais, podendo envolver, por exemplo, uma ou duas etapas. Um material comumente utilizado na preparação da casca em PQs é o sulfeto de zinco (ZnS), uma vez que além de possibilitar as melhorias nas propriedades dos materiais sintetizados, é tratado como um material de baixa toxicidade, possibilitando a obtenção de materiais ambientalmente mais amigáveis. ${ }^{54}$ A representação de um PQ com caroço de sulfeto de cobre e índio (CIS), e uma estrutura caroço/casca com casca de $\mathrm{ZnS}$, com o ligante de superfície TGA, são apresentadas na Figura 7.
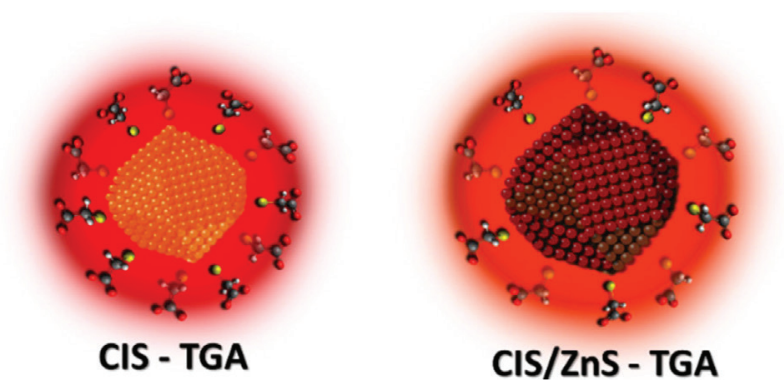

Figura 7. Representação ilustrativa de PQs caroço e caroço/casca, respectivamente, de CIS com o ligante TGA e camada de ZnS

Assim, neste trabalho é proposta a preparação e caracterização de pontos de quânticos de sulfeto de cobre, índio e zinco (CIZS), com uma camada de sulfeto de zinco ( $\mathrm{ZnS}$ ), em meio aquoso, utilizando glutationa reduzida como ligante de superfície, como uma aula experimental da disciplina "Química do Estado Sólido". A síntese utiliza uma metodologia simples e que possibilita obtenção de nanocristais coloidais de qualidade e com potencial para diversas aplicações.

O trabalho tem como objetivo a motivação de estudantes de graduação, principalmente em química, pelo contato com um experimento de obtenção de materiais de interesse tecnológico. Ressalta-se a dificuldade de contato e acesso da maioria dos estudantes com relação à síntese em sistemas coloidais e as caracterizações de materiais de interesse tecnológico, como os nanomateriais. O trabalho tem como intuito explorar com os alunos diversos conceitos, tanto novos quanto já abordados, como os conceitos envolvendo nanotecnologias, sínteses coloidais, estruturas de sólidos, caracterizações, entre diversos outros, possibilitando a aplicação dos conhecimentos adquiridos por meio de um contato mais direto com a pesquisa.

O procedimento descrito foi desenvolvido na disciplina "Química do Estado Sólido", na Universidade Federal de São João del-Rei, no ano de 2019, para alunos do $8^{\circ}$ período de graduação em química. Considera-se que o experimento seja mais adequado para a aplicação em períodos mais avançados do curso de química, de forma que possam ser utilizados, discutidos e aplicados os conhecimentos adquiridos durante o curso. Possibilitando ainda a oportunidade de explorar a interdisciplinaridade do assunto abordado.

\section{PARTE EXPERIMENTAL}

\section{Reagentes}

Os seguintes reagentes foram utilizados: cloreto de cobre (II) dihidratado (99\%), cloreto de índio (III) tetrahidratado (97\%), glutationa reduzida (98\%) (ou ácido mercaptopropiônico ou ácido tioglicólico) e sulfeto de sódio nonahidratado (99,9\%), obtidos da Sigma Aldrich. Cloreto de zinco (97\%) (Dinâmica), hidróxido de amônio (28 - 30\%) da Vetec e acetona $(99,75 \%)$ (êxodo científica).

\section{Caracterização}

Espectros de absorção dos nanomateriais sintetizados foram obtidos utilizando-se um espectrofotômetro de duplo feixe com um detector do tipo fotomultiplicador, modelo UV-2550 da Shimadzu. Medidas foram realizadas na região entre 900 e $200 \mathrm{~nm}$. Como solvente foi utilizada água deionizada e as medidas foram feitas utilizando-se cubetas de quartzo (Hellma), de $1 \mathrm{~cm}$ de caminho óptico.

Para as medidas referentes à emissão dos materiais, foi utilizado um espectrofluorímetro RF-5301PC, Shimadzu. Os espectros foram registrados na região de 420 a $780 \mathrm{~nm}$, sendo utilizadas cubeta de quartzo com $1 \mathrm{~cm}$ de caminho óptico, polida nas quatro faces. Foi utilizada uma lâmpada de xenônio de $150 \mathrm{~W}$, como fonte de excitação, sendo o comprimento de excitação utilizado fixado em $400 \mathrm{~nm}$. Observa-se que sendo o comprimento de onda de excitação de $400 \mathrm{~nm}$, a região de leitura foi de 420 a $780 \mathrm{~nm}$, de forma que não fossem registradas regiões de harmônicos referentes à fonte de excitação.

Difratogramas de raios $\mathrm{X}$ foram obtidos utilizando-se um difratômetro XRD-6000, Shimadzu, operando em modo de varredura de 10 a $70^{\circ}$, com radiação $\mathrm{k} \alpha_{\mathrm{Cu}}(\lambda=1,5406 \AA$ ) , gerada a $30 \mathrm{kV}$ e com corrente de $30 \mathrm{~mA}$. Passos de $0,02^{\circ}$ e tempo de amostragem de $2 \mathrm{~h}$ 
foram utilizados. Fendas de divergência de 1,0 mm, espalhamento de $1,0 \mathrm{~mm}$ e de recebimento de $0,3 \mathrm{~mm}$ foram utilizadas durante as análises. Foram registrados os padrões de difração das amostras de pontos quânticos pulverizadas em acetona, em porta amostra de silício não-reflexivo. O preparo das amostras foi feito por meio da precipitação, pela adição de acetona, centrifugação e secagem a $40^{\circ} \mathrm{C}$.

Espectros na região do infravermelho foram obtidos utilizando-se um espectrômetro infravermelho da Perkin Elmer, modelo Spectrum GX, com transformada de Fourier. Foram obtidos espectros na de região de 4000 a $400 \mathrm{~cm}^{-1}$, com resolução de $2 \mathrm{~cm}^{-1}$ e acúmulo de 32 espectros. As amostras foram preparadas pela dispersão em $\mathrm{KBr}$ e pastilhamento por meio de uma prensa hidráulica, com 10 toneladas de pressão para a compactação.

\section{Síntese dos pontos quânticos}

É importante destacar a imprescindibilidade dos cuidados com segurança, e, portanto, do uso de equipamentos de proteção, como avental, luvas e óculos de segurança, bem como o uso de capela, e ainda a cautela no manuseio dos equipamentos. Sugere-se a solicitação de preparo de pré-relatório, de forma que sejam discutidas características como, toxicidade dos reagentes e cuidados relacionados ao descarte, uma vez que é realizada a síntese de um nanomaterial. A síntese dos PQs foi realizada por meio de uma rota coloidal aquosa, direta e simples. Foram utilizados como precursores cloretos de cobre, índio e zinco, e sulfeto de sódio como fonte de enxofre. Proporção molar de $\mathrm{Cu}: \operatorname{In}: \mathrm{Zn}: \mathrm{S}$ de 1:3,75:1,25:10, foi utilizada para o preparo dos nanocristais, e uma proporção molar de $\mathrm{Cu}: \mathrm{Zn}$ de $1: 1$ foi utilizada no preparo da casca crescida, após o preparo do caroço, nos nanocristais.

As nanopartículas foram sintetizadas em soluções aquosas e estabilizadas por um ligante de superfície, tendo sido utilizada glutationa reduzida, sendo possível o uso de vários outros ligantes. Na Figura 8 é proposto um esquema das etapas experimentais utilizadas no experimento. Em um procedimento típico de síntese, são utilizados $10 \mathrm{~mL}$ de água, como solvente, sendo adicionados $40 \mu \mathrm{L}$ de uma solução aquosa de $\mathrm{CuCl}_{2} \cdot 2 \mathrm{H}_{2} \mathrm{O}\left(1 \mathrm{~mol} \mathrm{~L}^{-1}\right)$, seguidos por $150 \mu \mathrm{L}$ de uma solução aquosa de $\mathrm{InCl}_{3} \cdot 4 \mathrm{H}_{2} \mathrm{O}\left(1 \mathrm{~mol} \mathrm{~L}^{-1}\right)$ e $100 \mu \mathrm{L}$ de uma solução aquosa de $\mathrm{ZnCl}_{2}\left(0,5 \mathrm{~mol} \mathrm{~L}^{-1}\right)$ e então adicionados $0,9 \mathrm{mmol}$ do ligante de superfície. $\mathrm{O}$ pH da solução foi então ajustado para 10 , utilizando solução de $\mathrm{NH}_{4} \mathrm{OH}$ (30\%). Foram então adicionados 400 $\mu \mathrm{L}$ de solução aquosa de $\mathrm{Na}_{2} \mathrm{~S} .9 \mathrm{H}_{2} \mathrm{O}\left(1 \mathrm{~mol} \mathrm{~L}^{-1}\right)$. A solução foi então mantida a cerca de $100{ }^{\circ} \mathrm{C}$, em banho-maria, durante $1 \mathrm{~h}$.

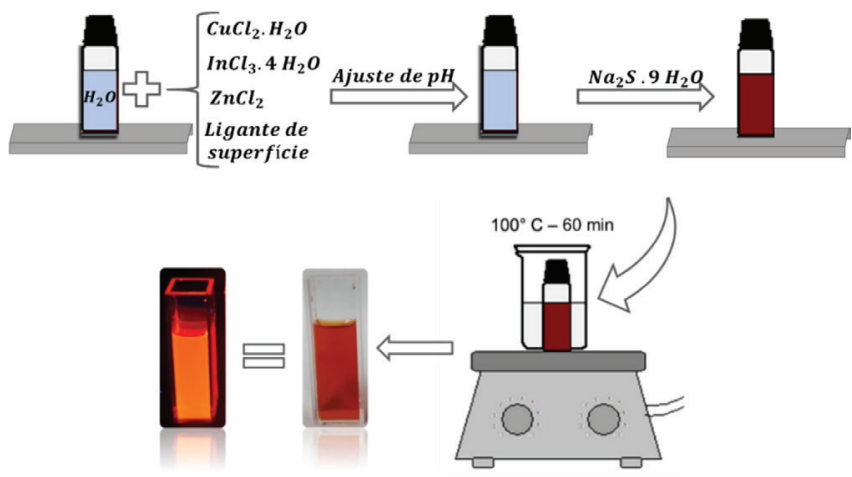

Figura 8. Esquema ilustrativo do procedimento de síntese dos PQs de CIZS

Após a síntese do caroço de CIZS, uma camada fina de ZnS (casca) é crescida nos nanocristais. O uso da casca para o encobrimento dos nanocristais é uma tática altamente utilizada, uma vez que o uso da estrutura caroço-casca possibilita a obtenção de materiais com características melhores. Para a adição da casca, $40 \mu \mathrm{L}$ de cloreto de zinco $\left(0,5 \mathrm{~mol} \mathrm{~L}^{-1}\right)$ foram adicionados a $5 \mathrm{~mL}$ da solução dos PQs, que foi mantida a cerca de $100{ }^{\circ} \mathrm{C}$, em banho-maria, durante 30 min (Figura 9). Como fonte de enxofre para a casca, os próprios nanocristais foram utilizados, de forma que somente íons de zinco foram adicionados à solução.

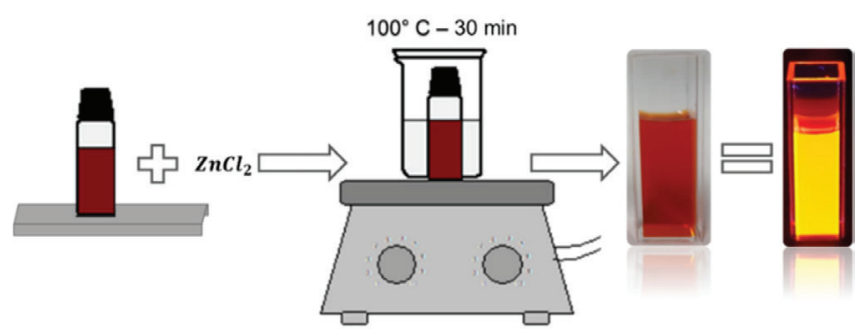

Figura 9. Esquema ilustrativo do procedimento de adição da casca de ZnS aos PQs de CIZS

\section{RESULTADOS E DISCUSSÕES}

A síntese de pontos quânticos quaternários ambientalmente amigáveis foi realizada com os estudantes do $8^{\circ}$ período do curso de graduação em química, na disciplina "Química do Estado Sólido". Uma rota de síntese simples e rápida, foi utilizada para obtenção dos nanocristais, baseada no uso de uma banho-maria e curtos tempos de sínteses. A síntese coloidal aquosa mostra-se uma rota interessante para produção dos PQs, possibilitando a obtenção de PQs de qualidade por um método simples, rápido e de fácil execução para um experimento.

A síntese dos PQs de CIZS e CIZS/ZnS foi realizada pelos estudantes com o ligante GSH. Ao final do procedimento uma alíquota de cada amostra foi retirada, para que fossem feitas as análises de absorção e de emissão. Ao restante do material foi adicionado acetona, para precipitação do material, que foi centrifugado e seco. Análises de absorção no UV-vis e de emissão das amostras, com e sem a casca, foram feitas pelos estudantes. O preparo das amostras para análise de difração de raios $\mathrm{X}$ e espectroscopia na região do infravermelho, bem como a realização da análise, foram acompanhados.

Os materiais sintetizados foram caracterizados óptica e estruturalmente pelas técnicas de espectroscopia de absorção UV-vis, fotoluminescência e de infravermelho, bem como difração de raios X. Os espectros de absorção e emissão estão apresentados na Figura 10. O material obtido diretamente da síntese apresentou fotoluminescência visual de cor laranja para o caroço e cor laranja com emissão visualmente mais intensa para o material após o crescimento da casca (apresentados nas Figura 8 e 9), quando excitadas por uma lâmpada UV com comprimento de onda de excitação de $365 \mathrm{~nm}$.
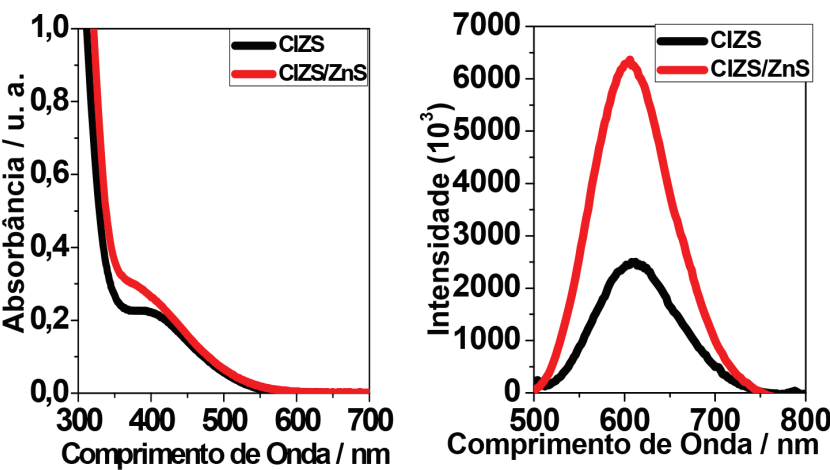

Figura 10. Espectros de absorção e emissão, respectivamente, para os $P Q s$ CIZS sintetizados, com e sem a adição da casca de ZnS 
Os espectros de absorção e emissão evidenciam a formação do material fotoluminescente, que absorve e emite na região do visível do espectro eletromagnético. O material mostra-se um bom absorvedor, absorvendo em uma ampla faixa do visível. Informações referentes à energia de banda proibida podem também ser obtidas por meio dos espectros de absorção dos materiais, e os dados obtidos estão apresentados na Tabela 1. A banda centralizada em $393 \mathrm{~nm}(3,15 \mathrm{eV})$ para o CIZS e em $376 \mathrm{~nm}(3,30 \mathrm{eV})$ para o CIZS/ZnS, é associada à primeira transição exitônica dos PQs. ${ }^{55}$ Os materiais apresentaram emissão na região do laranja, com máximos em torno de 603 e $608 \mathrm{~nm}$. O aumento da intensidade relativa de emissão indica a efetividade da formação da casca, pela melhoria da propriedade relativa à emissão dos nanocristais sintetizados, representadas também pelo rendimento quântico, apresentado na Tabela 1 , indicando a melhoria das propriedades de superfície dos materiais. Foi observado um aumento da capacidade de emissão para quase o dobro do rendimento quântico, pela adição da casca.

Tabela 1. Dados espectroscópicos referentes aos PQs sintetizados, CIZS e CIZS/ZnS

\begin{tabular}{lcccc}
\hline Amostra & $\begin{array}{c}\text { Máx. Abs } \\
(\mathrm{nm})\end{array}$ & Gap (eV) & $\begin{array}{c}\text { Máx. Em } \\
(\mathrm{nm})\end{array}$ & Фf $(\%)$ \\
\hline CIZS & 393 & 3,15 & 608 & 4 \\
CIZS/ZnS & 376 & 3,30 & 603 & 7 \\
\hline
\end{tabular}

A fim de obter mais informações a respeito da estrutura do material, sendo ainda uma forma de checar a formação do material de interesse, a técnica de difração de Raios X foi utilizada. Os difratogramas obtidos são apresentados na Figura 11.
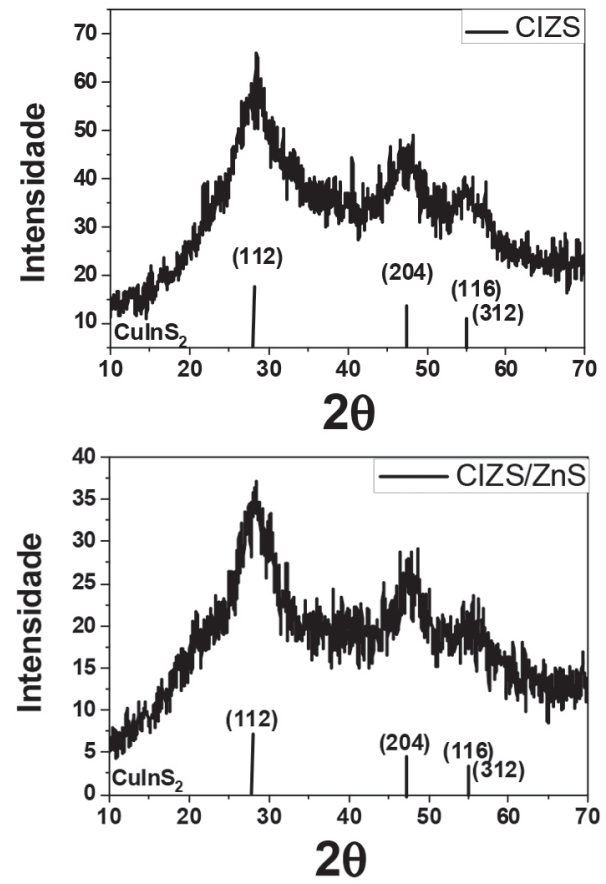

Figura 11. Difratogramas de raios $X$ das amostras de CIZS e CIZS/ZnS, respectivamente

A observação dos difratogramas obtidos mostra, para ambas as amostras, picos similares, alargados e de baixa intensidade (inflexões), o que é atribuído ao pequeno tamanho das partículas, em torno de $28^{\circ}$, $47^{\circ}$ e $55^{\circ}$, sendo os planos esperados (112), (204/220) e (116/312), indexados ao padrão de referência (JCDPS \#85-1575), ${ }^{56,57}$ referentes à fase tetragonal da calcopirita sulfeto de cobre e índio, possibilitando indicar a formação do material de interesse.
A inspeção dos espectros infravermelho dos materiais possibilita obtenção de informações a respeito da interação entre o ligante de superfície e os PQs. Os espectros do ligante de superfície GSH, bem como do material com esse ligante, são apresentados na Figura 12.

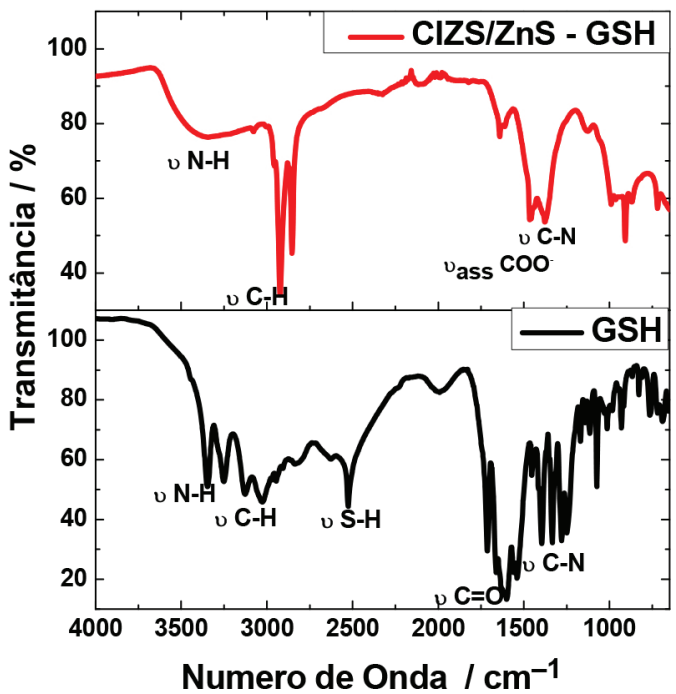

Figura 12. Espectros vibracionais na região do infravermelho do ligante de superficie GSH e dos PQs passivados com GSH

A análise das bandas apresentadas nos espectros na região do infravermelho obtidos mostram que os principais grupos funcionais do ligante GSH são encontrados, por meio de vibrações características como as referentes às bandas de carboxilato e amina, no entanto, não há o aparecimento da banda característica referente à ligação $\mathrm{S}-\mathrm{H}$, na região de $2550-2680 \mathrm{~cm}^{-1}$, o que é frequentemente atribuído à interação do enxofre com átomos dos PQs. ${ }^{43}$ Esse resultado mostra concordância com o pH do meio reacional, uma vez que em meio com pH superior aos Kas dos grupos é esperado que esses se encontrem desprotonados. Adicionalmente, sendo o enxofre menos eletronegativo que o oxigênio, tióis não fazem ligações de hidrogênio fortes e, consequentemente, têm atrações intermoleculares mais fracas; assim, os grandes íons tiolato não são muito bem solvatados em solventes próticos (como a água). Dessa forma, íons tiolato são bons nucleófilos, interagindo mais facilmente com ácidos moles, como o cátion de cobre, sendo possivelmente onde ocorre a interação com os PQs.

O experimento foi utilizado para trabalhar com os estudantes assuntos como síntese de nanopartículas, caracterização óptica, espectroscopias, caracterização estrutural e difração de raios X, proporção molar, ligantes de superfície, materiais semicondutores, rota de síntese coloidal, melhoria das propriedades pela adição de uma casca ao material, estrutura de sólidos cristalinos, transição eletrônica, entre outros assuntos. $\mathrm{O}$ experimento mostrou-se um interessante meio para introduzir aos alunos um material de grande interesse tecnológico, de uma forma simples e acessível, despertando o interesse dos estudantes ao mesmo tempo que possibilitava a aplicação prática dos conhecimentos já adquiridos ao longo do curso. Ao final do experimento, os alunos responderam a um questionário envolvendo o tema abordado. Esse questionário se encontra no Material Suplementar e possibilitou confirmar a motivação dos estudantes com relação a temas relacionados à nanotecnologia e aos nanomateriais.

\section{CONCLUSÕES}

Pontos quânticos ambientalmente amigáveis de sulfeto de cobre e índio foram sintetizados por uma rota de síntese "verde" e consideravelmente simples. Os pontos quânticos sintetizados 
em meio aquoso apresentam potencial para diversas aplicações, entre elas aplicações fotovoltaicas. Além disso, a síntese coloidal em meio aquoso mostrou-se como um método simples e rápido de obter PQs de qualidade.

A aplicação do experimento de síntese e caracterização de pontos quânticos ambientalmente amigáveis a estudantes da disciplina "Química do Estado Sólido", do curso de química, possibilitou o contato dos alunos com materiais de grande interesse tecnológico, sendo um material amplamente estudado por diversos grupos de pesquisa no mundo todo.

Diversos conceitos, como a síntese de nanopartículas luminescentes e algumas de suas caracterizações, entre muitos outros, puderam ser levados aos estudantes e usados na discussão de diversos tópicos de interesse na graduação do curso de química. A síntese foi executada pelos estudantes de forma simples e descomplicada, ajudando a desconstruir a ideia de alta complexidade envolvida na síntese de nanopartículas.

Outras técnicas de caracterização, bem como tópicos de ensino, poderiam ter sido usadas e abordadas durante o experimento, no entanto, a limitação de tempo, bem como de uma maior variedade de equipamentos, podem inviabilizar essa diversificação. O experimento é bastante atrativo, principalmente por se tratar do preparo de um material que apresenta luminescência na região do visível, quando exposto a radiação no ultravioleta. Tendo o experimento despertado o interesse dos estudantes, esse texto foi redigido com o intuito de estimular o acesso e contato dos discentes com a pesquisa.

\section{MATERIAL SUPLEMENTAR}

O questionário utilizado após a execução do experimento está disponível em http://quimicanova.sbq.org.br, na forma de arquivo PDF, com acesso livre.

\section{AGRADECIMENTOS}

Os autores agradecem à CAPES, ao CNPq, à FAPEMIG e à RQMG pelo apoio recebido.

\section{REFERÊNCIAS}

1. Andrews, D. L.; Lipson, R. H.; Nann, T.; Comprehensive nanoscience and nanotechnology, $2^{\mathrm{a}}$ ed., Elsevier: Amsterdam, 2019.

2. Ngo, T. T.; Mora-Seró, I.; J. Phys. Chem. Lett. 2019, 10, 1099.

3. Stroyuk, O.; Raevskaya, A.; Gaponik, N.; Chem. Soc. Rev. 2018, 47, 5354.

4. Hong, Y.; Wu, Y.; Wu, S.; Wang, X.; Zhang, J.; Isr. J. Chem. 2019, 59, 1.

5. Jain, S.; Bharti, S.; Bhullar, K. G.; Tripathi, K. S.; Journal of Luminescence 2019, doi: 10.1016/j.jlumin.2019.116912.

6. Alqahtani, F. S.; Farooq, A. W.; Ali, M. S.; Tawfik, W.; J. Optoelectron Adv. Mater. 2019, 21, 385 .

7. Jindal, S.; Giripunje, M. S.; Kondawar, B. S.; Koinkar, P.; J. Phys. Chem. Solids 2017, doi: 10.1016/j.jpcs.2017.11.026.

8. Silva, O. F.; Viol, S. C. L.; Ferreira, L. D.; Alves, J. L. A.; Schiavon, M. A.; Quim. Nova 2010, 33, 1933.

9. Klimov, V.I., Nanocrystal Quantum Dots $2^{\text {th }}$ ed., CRC: New York, 2010.

10. Altaf, T. Ç.; Sankir, D. N.; Int. J. Hydrogen Energy 2019, doi: 10.1016/j. ijhydene.2018.11.047

11. Guan, Z.; Chen, F.; Liu, Z.; Lv, P.; Chen, M.; Guo, M.; Li, X.; Teng, F.; Che, S.; Tang, A.; Org. Electron. 2019, doi: 10.1016/j.orgel.2019.06.024.

12. Zhang, R.; Deng, T.; Wang, J.; Wu, G.; Li, S.; Gu, Y.; Deng, D.; New J. Chem. 2019, doi: 10.1039/c7nj00573c.

13. Chen, L.; Xia, N.; Li, T.; Bai, Y.; Chen, X.; Microchim. Acta 2019, doi: 10.1007/s00604-016-1903-7.
14. Tang, X.; Yang, J.; Li, S.; Chen, W.; Hu, Z.; Qiu, J.; Front. Chem. 2019, doi: 10.3389/fchem.2019.00499.

15. Li, C.; Wu, P.; Luminescence 2019, doi: 10.1002/bio.3679.

16. Nong, J.; Lan, G.; Jin, W.; Luo, P.; Guo, C.; Tang, X.; Zang, Z.; Wei, W.; J. Mater. Chem. C 2019, doi: 10.1039/c9tc01395d.

17. Mansur, P. A. A.; Mansur, S. H.; Tabare, C.; Paiva, A.; Capanema, V. S. N.; J. Mater. Sci.: Mater. Electron. 2019, doi: 10.1007/s10854-01900719-0.

18. Altintas, Y.; Talpur, Y. M.; Unlu, M.; Mutlugun, E.; J. Phys. Chem. C 2016, doi: 10.1021/acs.jpcc.6b01977.

19. Senthilkumar, M., Mary, CI, Pandiyarajan, M.; Manobalaju, G.; Babu, M. S.; Bull. Mater. Sci. 2019, doi: 10.1007/s12034-019-1957-3.

20. Du, J.; Du, Z.; Hu, J.; Pan, Z.; Shen, Q.; Sun, J.; Long, D.; Dong, H.; Sun, L.; Zhong, X.; Wan, L.; J. Am. Chem. Soc. 2016, 138, 4201.

21. Jana, A.; Lawrence, N. K.; Teunis, B. M.; Mandal, M.; Kumbhar, A.; Sardar, R.; Chem. Mater. 2016, 28, 1107.

22. Gao, F.; Dai, H.; Pan, H.; Chen, Y.; Wang, J.; Chen, Z.; J. Colloid Interface Sci. 2018, doi: 10.1016/j.jcis.2017.11.066.

23. Yoon, S. Y.; Kim, J. H.; Jang, E. P.; Lee, S. H.; Jo, D. Y.; Kim, Y.; Do, Y. R.; Yang, H.; Chem. Mater. 2019, 31, 2627.

24. Jaffe, J. E.; Zunger, A.; Phys. Rev. B, 1983, 27, 5176.

25. Jaffe, J. E.; Zunger, A.; Phys. Rev. B, 1984, 29, 1882.

26. Stroyuk, O.; Weigert, F.; Raevskaya, A.; Spranger, F.; Würth, C.; Genger, U. R.; Gaponik, N.; Zahn, D. R. T.; J. Phys. Chem. C 2019, 123, 2632.

27. Vale, B. R. C.; Vieira, K. O.; Sousa, J. C. L.; Ferrari J. L.; Schiavon, M. A.; Quim. Nova 2015, 38, 22.

28. Azzellino, G.; Freyria, F. S.; Nasilowski, M.; Bawendi, M. G.; Bulovic, V.; Adv. Mater. Technol. 2019, 4, 1800727.

29. Stroyuk, O.; Raevskaya, A.; Spranger, F.; Gaponik, N.; Zahn, D. R. T.; ChemPhysChem 2019, doi:10.1002/cphc.201900088.

30. Hansen, E. C.; Bertram, S. N.; Yoo, J. J.; Bawendi, M. G.; Small 2019, 15,1901462

31. AbouElhamd, A. R.; Al-Sallal, K. A.; Hassan, A.; Energies 2019, 12 , 1058.

32. Guo, C.; Huang, Y.; Pan, Q.; Tao, T.; Li, F.; Zhang, Q.; Jin, X.; Li, Q.; Semicond. Sci. Technol. 2019, doi:10.1088/1361-6641/ab0314

33. Anand, A.; Zaffalon, M. L.; Gariano, G.; Camellini, A.; Gandini, M.; Brescia, R.; Capitani, C.; Bruni, F.; Pinchetti, V.; Zavelani-Rossi, M.; Meinardi, F.; Crooker, S. A.; Brovelli, S.; Adv. Funct. Mater. 2019, 29, 1906629.

34. Dutková, E.; Daneu, N.; Bujnáková, Z. L.; Baláž, M.; Kovác, J.; Kovác, J.; Baláž, P.; Molecules 2019, doi:10.3390/molecules24061031.

35. You, Y.; Tong, X.; Wang, W.; Sun, J.; Yu, P.; Ji, H.; Niu, X.; Wang, Z. M.; Adv. Sci. 2019, 6, 1801967.

36. Raevskaya, A.; Rosovik, O.; Kozytskiy, A.; Stroyuk, O.; Dzhagan, V.; Zahn, D. R.T.; RSC Adv. 2016, 6, 100145.

37. Zoroddua, M. A.; Aaseth, J.; Crisponid, G.; Medicia, S.; Peana, M.; Nurchid, V. M.; J. Inorg. Biochem. 2019, 195, 120.

38. Fu, Z.; Xi, S.; Toxicol. Mech. Methods 2019, 30, 167.

39. Goodman, C. H. L.; Douglas, R. W.; Physica 1954, 20, 1107.

40. Parthé, E.; Crystal Chemistry of Tetrahedral Structures, Gordon and Breach Science Publishers: New York, 1964.

41. Lakowicz, J. R.; Principles of Fluorescence Spectroscopy, Springer: New York, 2006.

42. Vaz, R.; Vieira, K. O.; Machado, C. E.; Ferrari, J. L.; Schiavon, M. A.; Quim. Nova 2015, 38, 1366.

43. Santos, C. I. L.; Carvalho, M. S.; Raphael, E.; Dantas, C.; Ferrari, J. L.; Schiavon, M. A.; Mater. Res. 2016; 19, 1407.

44. Pavia, D. L.; Lampman, G. M.; Kriz, G. S.; Vyvyan, J. R.; Introdução à espectroscopia, $4^{\text {th }}$ ed., Cengage Learning: São Paulo, 2010.

45. Weller, M. T.; Overton, T. L.; Rourke, J. P; Armstrong, F. A.; Química Inorgânica, $6^{\text {th }}$ ed., Bookman: Porto Alegre, 2017. 
46. Köhler, R.; Neumann, W.; Schmidbauer, M.; Hanke, M.; Grigoriev, D.; Schäfer, P.; Kirmse, H.; Häusler, I.; Schneide, R.; Nanosci. Technol. 2008 doi:10.1007/978-3-540-77899-8_5.

47. Farina, M.; Uma Introdução à Microscopia Eletrônica de Transmissão, $1^{\text {th }}$ ed., Livraria da Física: São Paulo, 2010.

48. Bruice, P. Y.; Química Orgânica. $4^{\text {th }}$ ed., Vol. 1, Pearson Prentice Hall: São Paulo, 2006.

49. Williams, A. T. R.; Winfield, S. A.; Miller, J. N.; Analyst 1983, 108, 1067.

50. Filho, P. C. S.; Serra, O. A.; Quim. Nova 2015, 38, 679.

51. Haoran, H.; Hamachi, L. S.; Rreza, I.; Wang, W.; Chem. Mater. (2019), doi: 10.1021/acs.chemmater.9b00971.
52. Tian, Y.; Xin, C.; Fang, Z.; Fang, X.; Zhou, J.; Yu, H.; Li, L.; Ju, Q.; Anal. Chim. Acta 2018, doi: 10.1016/j.aca.2018.09.043.

53. Wegner, K. D.; Dussert, F.; Truffier-Boutry, D.; Benayad, A.; Beal, D.; Mattera, L.; Reiss, P.; Front. Chem. 2019, doi:10.3389/ fchem.2019.00466.

54. Reiss, P.; Carrière, M.; Lincheneau, C.; Vaure, L.; Tamang, S.; Chem. Rev. 2016, 116, 10731.

55. Reis, G. B.; Rodriguez, R. D. F.; Santos, C. I. L.; Gontijo, L. A. P.; Schiavon, M. A.; Boni, L.; Mendonça, C. R.; Vivas, M. G.; Opt. Mater. 2018, 86,455 .

56. Liu, S.; Zhang, H.; Qia, Y.; Su, X.; RSC Adv. 2012, 2, 819.

57. Tang, A.; Hu, Z.; Yin, Z.; Ye, H.; Yang, C.; Teng, F.; Dalton Trans. 2015, 44,9251 . 